\title{
ETUDE COMPARÉE DES BILANS BIOLOGIQUES DE CERTAINS SOLS DE HÊTRAIE
}

\author{
F. TOUTAIN et Ph. DUCHAUFOUR \\ Centre de Pédologie biologique, \\ Centre national de la Recherche scientifique, 54 -Vandouvre
}

\section{RESUME}

La présente étude a pour objet d'effectuer un bilan des éléments nutritifs dans certaines Hêtraies acidiphiles de l'est de la France (deux hêtraies sur rendzine ont aussi été étudiées par comparaison). L'apport annuel par les litières de différents éléments est comparé à la quantité de ces éléments qui préexistent dans le sol sous forme mobilisable ; il est ainsi possible de mettre en évidence deux types de mobilisation pour les éléments ; 1) une mobilisation biologique (pour le carbone, l'azote et le calcium) aboutissant à un stockage de l'élément dans les horizons humifères, $A_{1}$ s'il s'agit d'un mull, $A_{0}$ s'il s'agit d'un mor ; 2) une mobilisation chimique (pour l'aluminium et le fer) aboutissant à une accumulation de l'élément dans les horizons minéraux par un processus d'altération. La silice et le phosphore, suivant les cas, participent aux deux types de mobilisation.

En définitive, la quantité d'éléments nutritifs, retournant annuellement au sol par la litière, ne subit que de faibles variations en comparaison avec la composition des roches mères et ne joue qu'un rôle mineur dans le déterminisme du type d'humus.

\section{INTRODUCTION}

Il est rare de voir, sous une essence donnée, le type d'humus varier autant que sous Hêtres. Dans les forêts de l'est de la France, sur calcaire bajocien, les forêts de Hêtres sont généralement des forêts à mull *. Dans les Vosges, et sur sols très acides,

* Les caractères des trois principaux ttypes d'humus formés en milieu aéré: mull, moder et mor, sont donnés dans le Précis de Pédologie de Ph. Duchaufour. Rappelons seulement que le mull est caractérisé par son activité microbiologique forte (décomposition rapide de la litière, d'où un horizon $A_{0}$ presque inexistant et un horizon $A_{1}$ plus ou moins épais avec un $\mathrm{C} / \mathrm{N}$ compris entre 10 et 20), que le mor au contraire présente une activité microbiologique très faible (d'où l'existence d'un horizon $A_{0}$ épais et d'un horizon $A_{1}$ très noir à $C / N$ toujours supérieur à 20). Quant au moder, il peut être considéré comme un type d'humus intermédiaire entre le mull et le mor et présente un horizon $A_{0}$ assez mince et un horizon $A_{1}$ assez épais à $C / N$ compris entre 15 et 25 . 
les types d'humus sont le plus souvent des moder et même quelquefois des mor, mais les Hêtraies à mull n’y sont pas rares. Il existe d’ailleurs dans la régions de Nancy, sur sable rhétien, des Hêtraies anciennes qui, tantôt sont des forêts à mull, avec une flore très caractéristique à Milium effusum et Melica uniflora, tantôt sont des forêts à moder ou à mor, avec des sols ocres podzoliques et même des sols podzoliques.

Il est certainement très important d'essayer de connaître les causes véritables du déterminisme de la formation de ces types d’humus car, si le matériel végétal, ici, permet de constituer soit un mull, soit un mor, le phénomène doit être général et doît permettre de comprendre quelles sont les conditions de formation de l'un ou l'autre de ces types d'humus.

Les calcaires et les roches mères riches en bases libèrent d'emblée une grande quantité de calcium qui sature les acides organiques, neutralise le milieu et améliore l'activité biologique ; il se forme alors un mull neutre ou basique, biologiquement toujours actif. Aussi, certains auteurs ont-ils pensé que, même en milieu acide, la formation d'un mull était liée à de forts apports annuels en cations (tout spécialement en (a) et en azote sous forme de litière, par rapport aux stations à humus brut où ces apports devaient être faibles. L'importance de l'azote dans les processus d'humification a été soulignée par JACQuin (1963), celle du calcium (et du pH) par Coulson et LEWIS (1960).

C'est ce que nous nous proposons de vérifier dans cette note. Pour reprendre le problème de façon générale, nous étudierons pour un certain nombre de stations de l'est de la France, les variations des teneurs des principaux constituants chimiques d'un horizon à l'autre, puis nous comparerons le bilan de ces éléments d'un profil à l'autre.

\section{I. - CHOIX DES STATIONS}

Le nombre des stations que nous avons retenues est de seize. Toutes ces stations sont sous Hêtres en fûtaie généralement pure ou en taches suffisamment importantes pour avoir pu contribuer à la formation d'un sol caractéristique. Les arbres y ont partout une densité suffisamment grande pour que la strate herbacée ait un développement pratiquement nul et que les matières premières qui tombent sur le sol soient uniquement constituées par les feuilles et débris de Hêtres. Toutes ces stations sauf une (la forêt de Fougères, en Ille-et-Vilaine) sont situées dans l'est de la France. Le forêt de Fougères, étudiée par nous antérieurement (Toutain, 1966), a été conservée, car elle permet de suivre une évolution du type d'humus (vers le type mor) et du type de sol (vers un micropodzol de surface) en fonction de l'âge du peuplement (fûtaie exploitée à 150 ans). Toutes ces stations ont été prises sur roche mère acide (alluvions anciennes, limons, sable, grès, schiste ou granite) à l'exception des deux stations à mull calcique de la Crédence et de Bellefontaine en forêt de Haye qui n'ont été retenues qu'à titre de comparaison. Cinq de ces stations sont des stations à mor et sont notées MO (il s'agit de mor de feuillus qui ne sont jamais aussi spectaculaires du point de vue morphologique, que les véritables humus bruts de résineux); cinq autres sont des stations à moder (notées mo), quatre ont un mull acide (mu), et deux sont des stations à mull saturé en calcium $(\mathrm{mc})$. 
Les types de sols sont résumés dans le tableau suivant (tableau 1) avec un classement qui correspond à des humus de plus en plus actifs.

TABLEAU 1

\begin{tabular}{|c|c|c|c|c|c|}
\hline Nom de la station & $\begin{array}{l}\text { Dési- } \\
\text { gnation }\end{array}$ & Altitude & $\begin{array}{c}\text { Type } \\
\text { d'humus }\end{array}$ & Roche mère & Type de sol \\
\hline La Costelle .... & MO1 & $700 \mathrm{~m}$ & Mor & Kératophyre & $\begin{array}{l}\text { Cryptopodzolique } \\
\text { lég' andosolique }\end{array}$ \\
\hline Basse Landzoll .. & $\mathrm{MO} 2$ & $700 \mathrm{~m}$ & Mor & $\begin{array}{l}\text { Schistes de } \\
\text { Villé }\end{array}$ & Cryptopodzolique \\
\hline Kligenthal $\ldots \ldots$ & $\mathrm{MO} 3$ & $850 \mathrm{~m}$ & Mor & Granite & Oere podzolique \\
\hline Fougères $\ldots \ldots$. & MO4 & $170 \mathrm{~m}$ & Mor & Limon & $\begin{array}{l}\text { Brun, légèrement les- } \\
\text { sivé à micropodzolisa- } \\
\text { tion de surface }\end{array}$ \\
\hline Lubine.. & MO5 & $620 \mathrm{~m}$ & Mor & $\begin{array}{l}\text { Schistes de } \\
\text { Villé }\end{array}$ & Brun acide \\
\hline Fossard I & mo1 & $780 \mathrm{~m}$ & Moder-Mor & Leptynite & Ocre podzolique \\
\hline Bezange $\ldots \ldots \ldots$ & $\mathrm{mo2}$ & $280 \mathrm{~m}$ & Moder-Mor & Grès rhétien & Ocre podzolique \\
\hline Harcotte $\ldots \ldots$. & $\mathrm{mo3}$ & $720 \mathrm{~m}$ & Moder & Granite & Cryptopodzolique \\
\hline Sainte-Hélène $\ldots$ & mo4 & $340 \mathrm{~m}$ & Moder & Limon & Lessivé à pseudogley \\
\hline Charmes...$\ldots$. & mo5 & $320 \mathrm{~m}$ & Moder & Limon & Lessivé à pseudogley \\
\hline Fossard II ..... & mu1 & $650 \mathrm{~m}$ & Mull-Moder & $\begin{array}{l}\text { Colluvium } \\
\text { Leptynite }\end{array}$ & Brun acide \\
\hline Hauts Bois .... & mu2 & $300 \mathrm{~m}$ & Mull acide & $\begin{array}{l}\text { Alluvions } \\
\text { anciennes }\end{array}$ & $\begin{array}{l}\text { Brun lessivé marmo- } \\
\text { risé }\end{array}$ \\
\hline Sainte-Marie .... & mu3 & $250 \mathrm{~m}$ & Mull acide & Grès rhétien & Brun acide \\
\hline Climont $\ldots \ldots \ldots$ & mu4 & $700 \mathrm{~m}$ & Mull acide & Colluvium grès & Brun acide \\
\hline Bellefontaine .... & $\mathrm{mc1}$ & $300 \mathrm{~m}$ & Mull calcique & $\begin{array}{l}\text { Calcaire bajo- } \\
\text { cien }\end{array}$ & R zndzine \\
\hline La Crédence .... & $\mathrm{mc} 2$ & $340 \mathrm{~m}$ & Mull calcique & Grouine & Rendzine \\
\hline
\end{tabular}

\section{II. - LES ELEMENTS DU BILAN}

\section{1. - Matériel et méthodes}

\section{1. Le matériel.}

Nous sommes parfaitement conscient du fait qu'il ne nous est pas possible, dans l'état actuel de nos connaissances, d'établir des bilans stricts pour chacune de ces stations, cela supposerait que nous connaissions les apports de cations dus par exemple aux eaux de pluie et au pluviolessivage des feuilles, les quantités d'azote dues à la nitrification microbiologique ou apportées par les micorrhizes, sans parler de ce que le sol reçoit d'exsudats racinaires. Les bilans que nous avons établis ici sont done très approchés, mais ils ont l'avantage de donner des ordres de grandeur; nous y avons distingué deux éléments principaux :

- les litières pour lesquelles nous avons établi le détail de la composition: de la litière annuelle $\left(\mathrm{L}_{1}\right)$, pour chacune des stations, qui représente en quelque sorte la recharge annuelle régulière et sûre en éléments chimiques; minéral ;

- de la litière ancienne $\left(\mathrm{L}_{22}\right)$ qui, elle, représente la réserve stockée au-dessus du sol

- les sols que nous avons restreints en épaisseur à la zone prospectée par les racines (observée sur le terrain) et où nous avons distingué : 
- les horizons organiques (que nous appellerons A) qui correspondent en fait aux horizons $\mathbf{A}_{0}+\mathbf{A}_{1}$, des sols à moder ou à mor, et à l'horizon $\mathrm{A}_{1}$ des sols à mull auquei s'ajoute souvent une partie de l'horizon $A_{1} B$. Ces horizons $A$ ont été facilement différenciés sur le terrain lorsqu'il s'agissait de sols à mor ou à moder (horizon noir bien tranché), mais nous n'avons pu les limiter, pour la plupart des sols à mull, qu'au vu des analyses chimiques (à une profondeur où a lieu une relative brisure du taux de matière organique dans le profil). L'épaisseur de ces horizons organiques varie de $5 \mathrm{~cm}$ pour la plupart des moders et des mors, à $20 \mathrm{~cm}$ pour la plus grande partie des sols à mull;

- les horizons minéraux (que nous appellerons B) qui correspondent au (B) d'altération cambique pour la plupart des sols, ou à la somme $\left(A_{2}+B\right)$ pour les sols qui ont des horizons minéraux plus différenciés en raison du lessivage.

\section{2. Les méthodes d'étude et d'analyses.}

Sur le terrain, ont été déterminées un certain nombre de données :

- l'apport annuel de litière $\mathrm{L}_{1}$ qui a été mesuré en récoltant la quantité de litière tombée fin octobre début novembre, sur des surfaces connues (cadre d'un mètre carré posé sur le sol) dans les différentes stations;

- la quantité de litière ancienne $L_{2}$ qui correspond aux débris de feuilles ramassées fin septembre sur le sol, sur des surfaces connues;

- l'épaisseur de la couche humifère $A_{0}$ comprise, pour les mors et pour les moders, entre cette litière ancienne et l'horizon $\mathrm{A}_{1}$;

- l'épaisseur de l'horizon $A_{1}$. La densité apparente de cet horizon a été déterminée en prélevant à ce niveau un cylindre de volume connu. Quant à la pierrosité, elle a été obtenue directement (rapport poids de cailloux d'un volume donné de sol) ;

$$
\text { poids total }
$$

- les caractéristiques principales des horizons (B) (densité apparente, pierrosité, et profondeur limitée à la zone prospectée par les racines et donc à l'épaisseur de terre entraînée par les chablis).

Au laboratoire ont été dosés :

- le carbone des litières et des sols, directement par pyrolyse (CARMOGRAPHE) ;

- l'azote des litières et des sols, par la méthode Kjeldahl ;

- les cations des litières, après passage au four à $700^{\circ}$ et fusion alcaline des cendres ; les éléments totaux comme $\mathrm{K}, \mathrm{Ca}, \mathrm{Mg}, \mathrm{Mn}$, ainsi que le fer, l'aluminium et la silice totale, ont été dosés par absorption atomique (TECHTRON). Quant au phosphore total, sa valeur a été déterminée par colorimétrie, après réduction par le chlorure stanneux du complexe phospho-molybdique ;

- les cations échangeables et la capacité totale d'échange des sols ont été dosés, après percolation sur le sol d'une solution d'acétate d'ammonium, par absorption atomique (TECHTRON) ;

- quant au fer, à l'aluminium et à la silice libres, leurs valeurs ont été obtenues par dosage au TECHTRON, après extraction au réactif combiné TAMM + hydrosulfite de sodium (méthode Duchaufour et Souchier) ;

- enfin, le phosphore dit assimilable est représenté par la somme du phosphore dosé après attaque en milieu acide (à $\left.\mathrm{H}_{2} \mathrm{SO}_{4} \mathrm{~N} / 250\right)$ et du phosphore extrait après attaque en milieu basique ( $\mathrm{NaOH} \mathrm{N/10)} \mathrm{(méthode} \mathrm{Duchaufour} \mathrm{et} \mathrm{Bonneau).}$

\section{2. - Les litières}

\subsection{Variations dans l'espace.}

— relatives à la quantité : un grand nombre de mesures faites en automne 1968 montrent que la quantité de litière varie relativement peu d'une station à l'autre (il 
s'agit de fûtaies presque toutes de même âge et de même état d'exploitation). La valeur moyenne se situe autour de 4 tonnes de feuilles sèches à l'hectare, les plus belles fûtaies ayant de l'ordre de 4,5 tonnes, et les forêts les plus dégradées (comme celle de La Costelle $\mathrm{MO}$ 1) ne recevant que 3 tonnes environ de litière fraîche chaque année ;

- relatives à la qualité : la composition des feuilles de Hêtre varie assez peu d'une station à l'autre. Les teneurs en carbone sont en moyenne de $50 \%$ avec des extrêmes de $44 \%$ pour Kligenthal (MO 3) et de $57 \%$ pour Harcotte (mo 3) ; les taux d'azote sont de l'ordre de $1 \%$ (chiffres extrêmes: $0,8 \%$ à La Costelle MO 1 et $1,2 \%$ à Kligenthal MO 3). Les apports correspondant à l'hectare (25 kg à La Costelle MO 1, et $35 \mathrm{~kg}$ au Climont mu 4) sont entre eux, pour cette station à mor et pour cette station à mull, dans le rapport de 1 à 1,4 . Par contre, les rapports de $\mathrm{C} / \mathrm{N}$ varient beaucoup plus (de 37 à Kligenthal MO 3 à 69 pour les Hauts-Bois mu 2) sans qu'il soit possible, semble-t-il, de rattacher cette variation à des caractères généraux de station. La teneur en potassium (avec des chiffres extrêmes de 0,30\% pour Charmes mo 5 et de $0,65 \%$ pour Fougères MO 4) varie du simple au double sans qu'il soit possible, là aussi, de rattacher cette variation à des caractères généraux de station; encore faudrait-il que les analyses de cet élément, facilement lessivable, aient été faites avec des conditions de prélèvements (date et pluviosité antérieures) comparables. Les teneurs en magnésium varient de 1 à $2(500 \mathrm{ppm}$ soit $0,05 \%$ pour Fossard I mo 1 et $1.000 \mathrm{ppm}$, soit $0,1 \%$ pour les deux stations sur calcaire). Par contre, les teneurs en calcium des litières ont une variabilité plus grande et sont très nettement liées aux teneurs en calcium de la roche mère (RüHL, 1957). La valeur la plus faible $(0,7 \%)$ correspond à la station de La Costelle (MO 1) qui n'a que 0,02 m.eq./ $100 \mathrm{~g}$ de sol de calcium échangeable dans l'horizon B, alors que La Crédence (mc 2), sur grouine calcaire, a une litière à 1,6\% de calcium. Sur l'ensemble des stations, les variations sont dans le rapport de 1 à 2,5 et sur l'ensemble des sols acides, elles sont de 1 à 2. Si nous comparons les apports annuels à l'hectare, ces apports ne varient d'une station à l'autre que de 1 à 2 ou au maximum à 3 , et ne reflètent donc pas les énormes variations de composition des roches mères en cet élément; ils ne peuvent donc jouer qu'un faible rôle dans le déterminisme des types d'humus. Les teneurs en silice se situent à 1,6\% à Sainte-Marie (mu 3) sur grès, et à $0,7 \%$ à Lubine (MO 5) sur schistes de Villé, le phosphore total variant entre $0,30 \%$ à Bezange (mo 2) et $0,11 \%$ à Charmes (mo 5) sans qu'il soit apparemment possible de trouver de lois générales.

\subsection{Variations dans le temps.}

Il s'agit là de voir quelles sont les transformations globales que subit la litière fraîche et, par conséquent, d'étudier l'état qualitatif et quantitatif de la litière ancienne :

- du point de vue quantitatif : de nombreuses mesures ont été faites sur le terrain, en septembre 1969. Leurs résultats figurent dans le tableau 2. Nous pouvons constater que les quantités à l'hectare de litière ancienne varient assez considérablement d'une station à l'autre, ce qui ne peut pas nous surprendre. Les chiffres extrêmes sont de $14 \mathrm{t} /$ ha pour Lubine (MO 5) où il y a une accumulation assez insolite de litière, à 
Bilan des

A. - Stations à mor

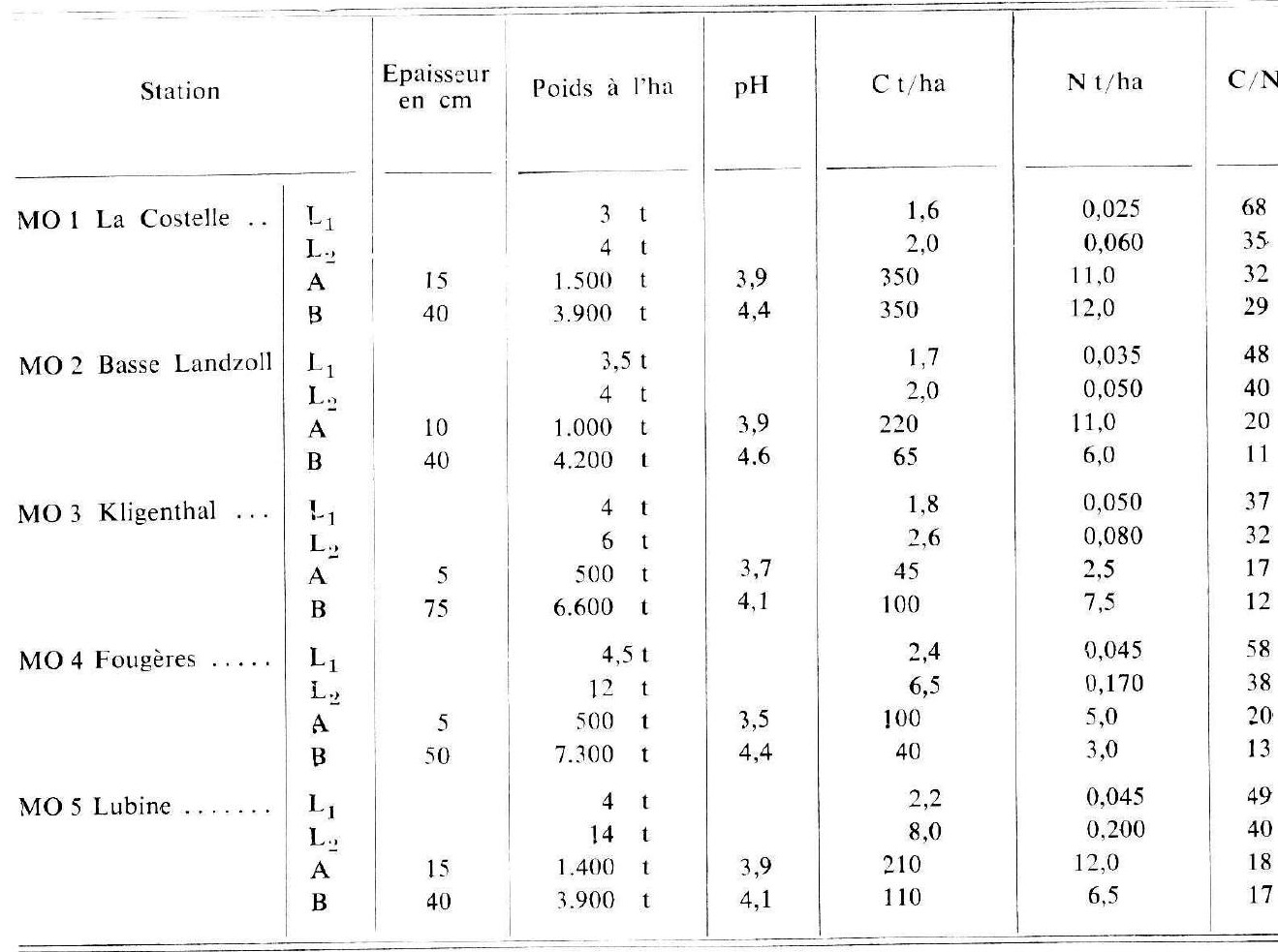

* Eléments totaux dans les feuilles, éléments libres dans les sols.

* $\mathrm{P}_{2} \mathrm{O}_{5}$ total dans les feuilles, $\mathrm{P}_{22} \mathrm{O}_{\overline{4}}$ assimilable dans les sols. 
(à lhectare)

\begin{tabular}{|c|c|c|c|c|c|c|c|c|c|}
\hline $\begin{array}{l}\mathrm{kg} / \mathrm{ha} \\
\text { chang. }\end{array}$ & $\begin{array}{l}\text { Ca kg/ha } \\
\text { échang. }\end{array}$ & $\begin{array}{l}\mathrm{Mg} \mathrm{kg} / \mathrm{ha} \\
\text { échang. }\end{array}$ & $\begin{array}{c}\mathrm{T} \\
\mathrm{m} . \mathrm{eq} / \\
100 \mathrm{~g}\end{array}$ & $\begin{array}{c}\mathrm{S} / \mathrm{T} \\
\text { en } \%\end{array}$ & $\mathrm{Fe} *$ & \multicolumn{2}{|c|}{$\mathrm{Al} *$} & $\mathrm{Si} *$ & $\begin{array}{l}\mathrm{P}_{2} \mathrm{O}_{5} \\
\mathrm{~kg} / \mathrm{ha}\end{array}$ \\
\hline 20 & 20 & 2 & & & $1 \mathrm{~kg}$ & & $\mathrm{~kg}$ & $30 \mathrm{~kg}$ & 7 \\
\hline 10 & 30 & 2 & & & $1 \mathrm{~kg}$ & & $\mathrm{~kg}$ & $50 \mathrm{~kg}$ & 10 \\
\hline 300 & 180 & 90 & 32 & 5 & $36 \mathrm{t}$ & 10 & & $4,5 \mathrm{t}$ & 220 \\
\hline 220 & 18 & 18 & 17 & 2 & $130 \mathrm{t}$ & 60 & & $50 \mathrm{t}$ & 800 \\
\hline 20 & 25 & 2 & & & $1 \mathrm{~kg}$ & 1 & & $30 \mathrm{~kg}$ & 6 \\
\hline 10 & 40 & 1 & & & $1 \mathrm{~kg}$ & 1 & kg & $40 \mathrm{~kg}$ & 10 \\
\hline 600 & 320 & 100 & 51 & 7 & 11 t & 5 & t & $0,2 \mathrm{t}$ & 200 \\
\hline 250 & 400 & 90 & 20 & 4 & $75 i$ & 40 & $\mathrm{t}$ & $1,7 \mathrm{t}$ & 700 \\
\hline 20 & 30 & 2 & & & $1 \mathrm{~kg}$ & & $\mathrm{~kg}$ & $30 \mathrm{~kg}$ & 8 \\
\hline 10 & 60 & 2 & & & $2 \mathrm{~kg}$ & & $\mathrm{~kg}$ & $60 \mathrm{~kg}$ & 15 \\
\hline 80 & 300 & 30 & 24 & 15 & $1.4 \mathrm{t}$ & 0 & & $0,2 \mathrm{t}$ & 70 \\
\hline 300 & 500 & 150 & 12 & 5 & $35 \mathrm{t}$ & 17 & $\mathrm{t}$ & $5 \mathrm{t}$ & 1.100 \\
\hline 30 & 20 & 3 & & & $1 \mathrm{~kg}$ & & $\mathrm{~kg}$ & $50 \mathrm{~kg}$ & 10 \\
\hline 25 & 80 & 5 & & & $4 \mathrm{~kg}$ & & & $180 \mathrm{~kg}$ & 40 \\
\hline 140 & 70 & 120 & 79 & 9 & $2 \mathrm{t}$ & 1, & & $0,2 t$ & 70 \\
\hline 250 & 700 & 140 & 15 & 5 & $75 \mathrm{t}$ & 30 & $\mathrm{t}$ & $0,7 \mathrm{t}$ & 1.000 \\
\hline 20 & 30 & 3 & & & $2 \mathrm{~kg}$ & 1 & $\mathrm{~kg}$ & $30 \mathrm{~kg}$ & 6 \\
\hline 30 & 150 & 10 & & & $7 \mathrm{~kg}$ & 6 & $\mathrm{~kg}$ & $140 \mathrm{~kg}$ & 35 \\
\hline 420 & 500 & 120 & 60 & 5,5 & $16 \mathrm{t}$ & 5 & $t$ & $1,4 \mathrm{t}$ & 320 \\
\hline 270 & 160 & 60 & 18 & 3 & $66 \mathrm{t}$ & 16 & $\mathrm{t}$ & $5 \mathrm{t}$ & 620 \\
\hline
\end{tabular}


TABL

Bilan des

B. - Stations à moder.

\begin{tabular}{|c|c|c|c|c|c|c|c|}
\hline \multicolumn{2}{|l|}{ Station } & \multirow[t]{3}{*}{$\begin{array}{l}\text { Epaisseur } \\
\text { en cm }\end{array}$} & \multirow{3}{*}{$\begin{array}{l}\text { Poids à l'ha } \\
\begin{array}{r}4 \mathrm{t} \\
8 \mathrm{t}\end{array}\end{array}$} & \multirow[t]{3}{*}{$\mathrm{pH}$} & \multirow{2}{*}{$\begin{array}{c}\mathrm{Ct} / \mathrm{ha} \\
2,0\end{array}$} & \multirow{2}{*}{$\begin{array}{c}N \mathrm{t} / \mathrm{ha} \\
0,040\end{array}$} & \multirow{2}{*}{$\begin{array}{r}\mathrm{C} / \mathrm{N} \\
51\end{array}$} \\
\hline mo 1 Fossard 1. & $\mathrm{~L}_{1}$ & & & & & & \\
\hline & $\mathrm{L}_{2}$ & & & & 4,2 & 0,100 & 42 \\
\hline & $\mathrm{A}$ & 5 & $500 \mathrm{t}$ & 3,7 & 40 & 1,7 & 23 \\
\hline & B & 45 & $3.400 \mathrm{t}$ & 4,5 & 75 & 3,8 & 20 \\
\hline mo 2 Bezange .. & $\mathrm{L}_{1}$ & & $4 t$ & & 1,9 & 0,045 & 44 \\
\hline & $\mathrm{L}_{2}$ & & $10 \mathrm{t}$ & & 5,0 & 0,140 & 36 \\
\hline & A & 10 & $1.000 \mathrm{t}$ & 3,7 & 70 & 3,8 & 19 \\
\hline & B & 50 & $6.500 \mathrm{t}$ & 3,6 & 55 & 3,5 & 16 \\
\hline mo 3 Harcotte .. & $\mathrm{L}_{1}$ & & $4 \mathrm{t}$ & & 2,3 & 0,035 & 63 \\
\hline & $\mathrm{L}_{2}$ & & $6 \mathrm{t}$ & & 3,5 & 0,080 & 44 \\
\hline & A & 25 & $2.000 \mathrm{t}$ & 3,9 & 210 & 12,0 & 17 \\
\hline & B & 20 & $1.500 \mathrm{t}$ & 4,2 & 60 & 4,0 & 15 \\
\hline mo 4 Ste-Hélène & $\mathrm{L}_{1}$ & & $4 \mathrm{t}$ & & 2,0 & 0,035 & 54 \\
\hline & $\mathrm{L}_{2}$ & & $6 \mathrm{t}$ & & 3,0 & 0,080 & 39 \\
\hline & $A$ & 5 & $600 \mathrm{t}$ & 3,8 & 100 & 4,5 & 22 \\
\hline & B & 50 & $7.500 \mathrm{t}$ & 4,2 & 40 & 4,0 & 10 \\
\hline mo 5 Charmes .. & $\mathrm{L}_{1}$ & & $4 \mathrm{t}$ & & 2,0 & 0,035 & 53 \\
\hline & $\mathrm{L}_{2}$ & & $6 \mathrm{t}$ & & 3,0 & 0,080 & 37 \\
\hline & A & 5 & $600 \mathrm{t}$ & 4,1 & 150 & 6,0 & 26 \\
\hline & B & 40 & $6.000 \mathrm{t}$ & 4,3 & 7,0 & 0,3 & 23 \\
\hline
\end{tabular}

* Eléments totaux dans les feuilles, éléments libres dans les sols.

** $\mathrm{P}_{2} \mathrm{O}_{5}$ total dans les feuilles, $\mathrm{P}_{2} \mathrm{O}_{5}$ assimilable dans les sols. 
suite)

(à l'hectare)

\begin{tabular}{|c|c|c|c|c|c|c|c|c|c|}
\hline $\begin{array}{l}\mathrm{kg} / \mathrm{ha} \\
\text { chang. }\end{array}$ & $\begin{array}{c}\mathrm{Ca} \mathrm{kg/ha} \\
\text { échang. }\end{array}$ & $\begin{array}{l}\mathrm{Mg} \mathrm{kg} / \mathrm{ha} \\
\text { échang. }\end{array}$ & $\begin{array}{c}\mathrm{T} \\
\mathrm{m} . \mathrm{eq} / \\
100 \mathrm{~g}\end{array}$ & $\begin{array}{c}\mathrm{S} / \mathrm{T} \\
\text { en \% }\end{array}$ & $\mathrm{Fe} *$ & $\mathrm{Al}$ * & & $\mathrm{Si}$ * & $\underset{\mathrm{kg} / \mathrm{ha}}{\mathrm{P}_{2} \mathrm{O}_{5}}$ \\
\hline 20 & 30 & 2 & & & $1 \mathrm{~kg}$ & $1 \mathrm{~kg}$ & & $40 \mathrm{~kg}$ & 10 \\
\hline 20 & 80 & 3 & & & $3 \mathrm{~kg}$ & $3 \mathrm{~kg}$ & & $100 \mathrm{~kg}$ & 20 \\
\hline 160 & 120 & 30 & 32 & 8 & $2,2 \mathrm{t}$ & 0,7 & $\mathrm{t}$ & $0,5 \mathrm{t}$ & 40 \\
\hline 100 & 280 & 60 & 10 & 6 & $30 \mathrm{t}$ & 15 & $\mathrm{t}$ & $9 \mathrm{t}$ & 500 \\
\hline 20 & 40 & 2 & & & $2 \mathrm{~kg}$ & $1 \mathrm{~kg}$ & & $60 \mathrm{~kg}$ & 10 \\
\hline 20 & 130 & 4 & & & $6 \mathrm{~kg}$ & $4 \mathrm{~kg}$ & & $200 \mathrm{~kg}$ & 50 \\
\hline 80 & 250 & 25 & 11 & 15 & $1,3 \mathrm{t}$ & 0,33 & & $0,2 \mathrm{t}$ & 40 \\
\hline 300 & 70 & 50 & 5 & 5 & $18 \mathrm{t}$ & 6 & $\mathrm{t}$ & $2,5 \mathrm{t}$ & 450 \\
\hline 25 & 30 & 3 & & & $1 \mathrm{~kg}$ & $1 \mathrm{~kg}$ & & $30 \mathrm{~kg}$ & 10 \\
\hline 10 & 60 & 3 & & & $2 \mathrm{~kg}$ & $2 \mathrm{~kg}$ & & $70 \mathrm{~kg}$ & 20 \\
\hline 100 & 140 & 40 & 20 & 3 & $24 \quad t$ & 23 & $\mathrm{t}$ & $4 \mathrm{t}$ & 1.300 \\
\hline 50 & 130 & 25 & 18 & 4 & $20 \mathrm{t}$ & 18 & $\mathrm{t}$ & $3 \mathrm{t}$ & 1.200 \\
\hline 20 & 40 & 2 & & & $1 \mathrm{~kg}$ & $1 \mathrm{~kg}$ & & $50 \mathrm{~kg}$ & 10 \\
\hline 10 & 80 & 2 & & & $2 \mathrm{~kg}$ & $2 \mathrm{~kg}$ & & $70 \mathrm{~kg}$ & 20 \\
\hline 30 & 200 & 60 & 26 & 10 & $4 \mathrm{t}$ & 1,8 & $t$ & & 60 \\
\hline 80 & 400 & 100 & 6 & 10 & $120 \mathrm{t}$ & 20 & $\mathrm{t}$ & & 700 \\
\hline 10 & 50 & 2 & & & $1 \mathrm{~kg}$ & $1 \mathrm{~kg}$ & & $50 \mathrm{~kg}$ & 5 \\
\hline 6 & 100 & 2 & & & $2 \mathrm{~kg}$ & $2 \mathrm{~kg}$ & & $100 \mathrm{~kg}$ & 10 \\
\hline 100 & 200 & 80 & 17 & 19 & $5 \mathrm{t}$ & 1 & $\mathrm{t}$ & & 60 \\
\hline 500 & 1.200 & 500 & 11 & 18 & 80 & 20 & $\mathrm{t}$ & & 240 \\
\hline
\end{tabular}


TABL

Bilan des

C. - Stations à mull acide

\begin{tabular}{|c|c|c|c|c|c|c|c|}
\hline \multicolumn{2}{|l|}{ Station } & $\begin{array}{l}\text { Epaisseur } \\
\text { en cm }\end{array}$ & Poids à l'ha & $\mathrm{pH}$ & $\mathrm{Ct} / \mathrm{ha}$ & $\mathrm{N}$ t/ha & $\mathrm{C} / \mathrm{N}$ \\
\hline mu. 1 Fossard II & $\begin{array}{l}\mathrm{L}_{1} \\
\mathrm{~L}_{2 .} \\
\mathrm{A} \\
\mathrm{B}\end{array}$ & $\begin{array}{l}10 \\
50\end{array}$ & $\begin{array}{r}4 \mathrm{t} \\
3 \mathrm{t} \\
1.000 \mathrm{t} \\
4.000 \mathrm{t}\end{array}$ & $\begin{array}{l}3,6 \\
4,2\end{array}$ & $\begin{array}{r}2,0 \\
1,5 \\
70 \\
100\end{array}$ & $\begin{array}{l}0,045 \\
0,040 \\
4,0 \\
6,0\end{array}$ & $\begin{array}{l}46 \\
37 \\
19 \\
17\end{array}$ \\
\hline $\begin{array}{c}\text { mu. } 2 \text { Les Hauts- } \\
\text { Bois } \ldots \ldots \ldots . . .\end{array}$ & $\begin{array}{l}\mathrm{L}_{1} \\
\mathrm{~L}_{2} \\
\mathrm{~A} \\
\mathrm{~B}\end{array}$ & $\begin{array}{c}5 \\
40\end{array}$ & $\begin{array}{r}4 \mathrm{t} \\
2 \mathrm{t} \\
500 \mathrm{t} \\
4.200 \mathrm{t}\end{array}$ & $\begin{array}{l}4,8 \\
4,5\end{array}$ & $\begin{array}{l}2,1 \\
1,0 \\
40 \\
30\end{array}$ & $\begin{array}{l}0,035 \\
0,020 \\
2,5 \\
3,0\end{array}$ & $\begin{array}{l}69 \\
50 \\
16 \\
10\end{array}$ \\
\hline mu. 3 Sie-Marie. & $\begin{array}{l}\mathrm{L}_{1} \\
\mathrm{~L}_{.2} \\
\mathrm{~A} \\
\mathrm{~B}\end{array}$ & $\begin{array}{l}15 \\
50\end{array}$ & $\begin{array}{r}4 \mathrm{t} \\
3 \mathrm{t} \\
1.500 \mathrm{t} \\
6.200 \mathrm{t}\end{array}$ & $\begin{array}{l}4,5 \\
4,2\end{array}$ & $\begin{array}{l}2,0 \\
1,6 \\
62 \\
40\end{array}$ & $\begin{array}{l}0,040 \\
0,040 \\
4,0 \\
3,2\end{array}$ & $\begin{array}{l}50 \\
40 \\
16 \\
12\end{array}$ \\
\hline mu. 4 Le Climon: & $\begin{array}{l}\mathrm{L}_{1} \\
\mathrm{~L}_{2} \\
\mathrm{~A} \\
\mathrm{~B}\end{array}$ & $\begin{array}{l}20 \\
40\end{array}$ & $\begin{aligned} 3,5 \mathrm{t} \\
2,0 \mathrm{t} \\
2.200 \mathrm{t} \\
4.000 \mathrm{t}\end{aligned}$ & $\begin{array}{l}5,3 \\
5,2\end{array}$ & $\begin{array}{l}2,1 \\
1,0 \\
60 \\
60\end{array}$ & $\begin{array}{l}0,035 \\
0,025 \\
3,7 \\
4,4\end{array}$ & $\begin{array}{l}57 \\
40 \\
16 \\
13\end{array}$ \\
\hline
\end{tabular}

D. - Stations à mull calcique.

\begin{tabular}{|c|c|c|c|c|c|c|c|}
\hline \multicolumn{2}{|l|}{ Station } & \multirow[t]{3}{*}{$\begin{array}{l}\text { Epaisseur } \\
\text { en } \mathrm{cm}\end{array}$} & \multirow{2}{*}{$\begin{array}{c}\text { Poids à l'ha } \\
4,0 \\
4\end{array}$} & $\mathrm{pH}$ & $\mathrm{Ct} / \mathrm{ha}$ & $\mathrm{N} \mathrm{t} / \mathrm{ha}$ & \multirow{2}{*}{$\begin{array}{c}\mathrm{C} / \mathrm{N} \\
56\end{array}$} \\
\hline $\begin{array}{c}\text { mc. } 1 \text { Bellefontai- } \\
\text { ne } \ldots \ldots \ldots \ldots\end{array}$ & $\mathrm{L}_{1}$ & & & & 2,0 & 0,035 & \\
\hline & $\mathrm{L}_{2}$ & & 3,0 & & 1,6 & 0,035 & 46 \\
\hline & A & 5 & 500 & 7,5 & 80 & 5,0 & 15 \\
\hline & $\mathrm{B}$ & 25 & 2.100 & 7,7 & 50 & 4,3 & 12 \\
\hline mc. 2 la Crédence & $\mathrm{L}_{1}$ & & 4,5 & & 2,3 & 0,035 & 63 \\
\hline & $\mathrm{L}_{2}$ & & 4,0 & & 2,1 & 0,050 & 42 \\
\hline & A & 10 & 1.100 & 7,5 & 80 & 4,4 & 18 \\
\hline & B & 30 & 2.700 & 7,8 & 70 & 7,0 & 10 \\
\hline
\end{tabular}

* Eléments totaux dans les feuilles, éléments libres dans les sols. ** $\mathrm{P}_{2} \mathrm{O}_{5}$ total dans les feuilles, $\mathrm{P}_{2} \mathrm{O}_{5}$ assimilable dans les sols. 
uite)

(à l'hectare)

\begin{tabular}{|c|c|c|c|c|c|c|c|c|}
\hline $\begin{array}{l}\mathrm{kg} / \mathrm{ha} \\
\text { chang. }\end{array}$ & $\begin{array}{l}\mathrm{Ca} \mathrm{kg} / \mathrm{ha} \\
\text { échang. }\end{array}$ & $\begin{array}{l}\mathrm{Mg} \mathrm{kg} / \mathrm{ha} \\
\text { échang. }\end{array}$ & $\begin{array}{c}\mathrm{T} \\
\mathrm{m} . \mathbf{e q} / \\
100 \mathrm{~g}\end{array}$ & $\begin{array}{l}\mathrm{S} / \mathrm{T} \\
\text { en } \%\end{array}$ & $\mathrm{Fe}^{*}$ & $\mathrm{Al} *$ & $\mathrm{Si} *$ & $\underset{\mathrm{kg} / \mathrm{ha}}{\mathrm{P}_{2} \mathrm{O}_{5}}$ \\
\hline 20 & 30 & 2 & & & $2 \mathrm{~kg}$ & $1 \mathrm{~kg}$ & $50 \mathrm{~kg}$ & 10 \\
\hline 6 & 30 & 2 & & & $1 \mathrm{~kg}$ & $1 \mathrm{~kg}$ & $50 \mathrm{~kg}$ & 8 \\
\hline 230 & 200 & 60 & 25 & 8 & & & & 100 \\
\hline 180 & 240 & 100 & 10 & 7 & & & & 140 \\
\hline 20 & 40 & 2 & & & $1 \mathrm{~kg}$ & $1 \mathrm{~kg}$ & $60 \mathrm{~kg}$ & 10 \\
\hline 4 & 30 & 1 & & & $1 \mathrm{~kg}$ & $1 \mathrm{~kg}$ & $40 \mathrm{~kg}$ & 8 \\
\hline 100 & 250 & 50 & 15 & 26 & $8 \mathrm{t}$ & $0,8 \mathrm{t}$ & & 70 \\
\hline 500 & 500 & 600 & 18 & 34 & $80 \mathrm{t}$ & $8 \mathrm{t}$ & & 500 \\
\hline 20 & 40 & 3 & & & $2 \mathrm{~kg}$ & $2 \mathrm{~kg}$ & $65 \mathrm{~kg}$ & 10 \\
\hline 6 & 40 & 2 & & & $2 \mathrm{~kg}$ & $2 \mathrm{~kg}$ & $60 \mathrm{~kg}$ & 12 \\
\hline 120 & 840 & 55 & 7 & 45 & $7,5 \mathrm{t}$ & $2,3 \mathrm{t}$ & $0,8 \mathrm{t}$ & 180 \\
\hline 110 & 60 & 40 & 3 & 5 & $36 \mathrm{t}$ & $10 \mathrm{t}$ & $2,2 \mathrm{t}$ & 550 \\
\hline 20 & 25 & 2 & & & $1 \mathrm{~kg}$ & $1 \mathrm{~kg}$ & $30 \mathrm{~kg}$ & 6 \\
\hline 4 & 30 & 1 & & & $1 \mathrm{~kg}$ & $1 \mathrm{~kg}$ & $40 \mathrm{~kg}$ & 5 \\
\hline 170 & 200 & 80 & 6 & 16 & $8 \mathrm{t}$ & $3 \mathrm{t}$ & $4 \mathrm{t}$ & 200 \\
\hline 140 & 230 & 130 & 4 & 130 & $12 \mathrm{t}$ & $4 \mathrm{t}$ & 5 & 320 \\
\hline
\end{tabular}

\begin{tabular}{|c|c|c|c|c|c|c|c|c|}
\hline $\begin{array}{l}\mathrm{kg} / \mathrm{ha} \\
\text { hang. }\end{array}$ & $\begin{array}{l}\mathrm{Ca} \mathrm{kg} / \mathrm{ha} \\
\text { échang. }\end{array}$ & $\begin{array}{l}\mathrm{Mg} \mathrm{kg} / \mathrm{ha} \\
\text { échang. }\end{array}$ & $\begin{array}{c}\mathrm{T} \\
\mathrm{m} . \mathrm{eq} / \\
100 \mathrm{~g}\end{array}$ & $\begin{array}{l}\mathrm{S} / \mathrm{T} \\
\text { en } \%\end{array}$ & $\mathrm{Fe} *$ & $\mathrm{Al}$ * & $\mathrm{Si} *$ & $\begin{array}{c}\mathrm{P}_{2} \mathrm{O}_{5} * * \\
\mathrm{~kg} / \mathrm{ha}\end{array}$ \\
\hline 20 & 50 & 4 & & & $2 \mathrm{~kg}$ & $1 \mathrm{~kg}$ & $40 \mathrm{~kg}$ & 7 \\
\hline 6 & 50 & 2 & & & $2 \mathrm{~kg}$ & $1 \mathrm{~kg}$ & $40 \mathrm{~kg}$ & 8 \\
\hline 200 & 50.000 & 80 & 60 & 100 & $3,2 \mathrm{t}$ & & & 70 \\
\hline 250 & 200.000 & 800 & 10 & 100 & $7,0 \mathrm{t}$ & & & 100 \\
\hline 20 & 70 & 5 & & & $2 \mathrm{~kg}$ & $1 \mathrm{~kg}$ & $50 \mathrm{~kg}$ & 7 \\
\hline 8 & 80 & 3 & & & $2 \mathrm{~kg}$ & $2 \mathrm{~kg}$ & $50 \mathrm{~kg}$ & 9 \\
\hline 400 & 30.000 & 150 & 48 & 100 & & & & 50 \\
\hline 60 & 200.000 & 130 & 25 & 100 & & & & 100 \\
\hline
\end{tabular}


2 tonnes pour les stations à mull acide des Hauts Bois (mu 2) et du Climont (mu 3). Etant donné que les apports de litière annuelle sont tous du même ordre de grandeur, la quantité de litière ancienne présente dans chaque station est liée au type d'humus et est évidemment plus grande pour les mor que pour les mull. Deux ou trois stations pourtant échappent apparemment à ce raisonnement, mais ces exceptions peuvent être expliquées par des considérations topographiques (stations à forte pente ou fortement ventées). Ces chiffres permettent de définir une sorte de vie moyenne de la litière qui est d'un an et demi aux Hauts Bois (mu 2) et au Climont (mu 4) (puisqu'il reste encore 2 tonnes de litière avant la chute des feuilles), de l'ordre de 5 ans à Lubine (MO 5) et en moyenne de 2 à 3 ans pour les stations à moder ;

— du point de vue qualitatif: les teneurs en carbone des litières anciennes sont assez semblables à celles de la litière fraîche et sont assez homogènes (de 40 à $50 \%$ ) ; les chiffres obtenus pour l'azote sont aussi plus homogènes (de 1,5 à 2,0 \%) et plus élevés que pour la litière fraîche, ce qui donne des valeurs de $\mathrm{C} / \mathrm{N}$ plus faibles que précédemment, mais tout aussi imprévisibles. Les quantités de potassium se situent presque toutes autour de $0,20 \%$ et ont donc nettement diminué par rapport à celles de la litière fraîche, ce qui était prévisible, étant donné la facilité avec laquelle le potassium est entraîné. Les teneurs en calcium ont par contre beaucoup augmenté ; cette augmentation est, bien sûr, relative puisqu'elle est alimentée par les teneurs en calcium du «squelette» de la matière organique qui a été minéralisée. Les chiffres extrêmes se situent autour de $0,70 \%$ pour la Costelle (MO 1) et de 2,0 \% pour La Crédence ( $\mathrm{mc} 2$ ), l'ordre de décroissance des teneurs en calcium restant à peu près le même que précédemment. Les chiffres correspondant au magnésium présentent une diminution moyenne de l'ordre de 1,2 à 1,5 par rapport à ceux de la litière fraîche $(300 \mathrm{ppm}$, soit $0,03 \%$ pour Fossard I (mo 1) et 700 ppm, soit $0,07 \%$ pour Bellefontaine (mc 1)). Les teneurs relatives en silice passent en moyenne à $1,5 \%$ (avec comme chiffres extrêmes Sainte-Marie (mu 3) : $2 \%$ et Lubine (MO 5) : $1 \%$ ). Cette concentration relative s'observe aussi pour le phosphore : $0,50 \%$ pour Bezange (mo 2) et 0,17 \% pour la station de Charmes (mo 5).

\section{3. - Caractéristiques des sols}

— Vues sous l'angle quantitatif : chaque station est caractérisée dans notre étude par un poids de sol, rapporté à l'unité de surface et exprimé en tonne/hectare pour un horizon donné, mais aussi, et cela pour comparer les horizons entre eux, en tonne par $\mathrm{cm}$ de terre à l'hectare, soit, ce qui revient au même, en tonne pour $100 \mathrm{~m}^{3} \mathrm{de}$ sol.

L'épaisseur des horizons (A) (tels que nous les avons définis précédemment) est généralement faible. Elle varie entre 5 et $15 \mathrm{~cm}$ pour les mor, 5 et $10 \mathrm{~cm}$ pour les moder (à l'exception du sol de Harcotte (mo 3), qui a une morphologie très spéciale et où nous avons dû prendre $25 \mathrm{~cm}$ comme épaisseur de l'horizon $\mathrm{A}$ ) et entre 5 et $20 \mathrm{~cm}$ pour les mull. Les densités apparentes varient, dans cet horizon, entre 1,0 et 1,2 $\left(A_{0}+A_{1}\right.$ ou $A_{1}$ seul) et la pierrosité ne dépasse en général pas $10 \%$. Le poids de terre correspondant se situe entre 500 et $2200 \mathrm{t} /$ ha et 80 et $120 \mathrm{t} /$ ha pour $1 \mathrm{~cm} \mathrm{de} \mathrm{sol}$. 
L'épaisseur des horizons minéraux (B) sous-jacents varie entre $20 \mathrm{~cm}$ et $75 \mathrm{~cm}$, les densités apparentes se situent autour de 1,3 (entre 1,1 et 1,5 ) et la pierrosité y est très variable (elle varie entre 0 et $50 \%$ de cailloux) ; les poids de terre correspondant oscillent entre $1500 \mathrm{t}$ et $7500 \mathrm{t} /$ ha et entre $75 \mathrm{t}$ et $150 \mathrm{t} / \mathrm{ha}$ par $\mathrm{cm}$ de sol, avec une moyenne de $100 \mathrm{t} /$ ha par $\mathrm{cm}$ de sol.

- Vues sous l'angle qualitatif : à l'exception des deux stations reposant sur roche mère calcaire, dont les $\mathrm{pH}$ sont supérieurs à 7 , les $\mathrm{pH}$ des différents horizons de toutes ces stations sont généralement bas et inférieurs à 5. Les quantités de carbone et d'azote sont habituellement fortes dans les horizons superficiels de ces sols acides dont les $\mathrm{C} / \mathrm{N}$ sont souvent supérieurs à 20 (sauf pour les sols à mull). Dans les horizons (B), les valeurs de $\mathrm{S}$ sont faibles et les $\mathrm{S} / \mathrm{T}$ sont presque tous inférieurs à $20 \%$ (à l'exception évidente des sols sur roche mère calcaire). Les quantités de sesquioxydes libres sont très variables; quant à celles qui correspondent à l'acide phosphorique assimilable, elles se situent entre 100 et $250 \mathrm{ppm}$.

\section{ET D'UNE STATION A L'AUTRE \\ 1. - Le problème de la mobilisation biologique et de la mobilisation chimique des sols}

III. - COMPARAISON DES ELEMENTS DU BILAN D'UN HORIZON A L'AUTRE

Au cours de la pédogénèse, le sol s'enrichit progressivement en éléments libres ou échangeables, par rapport aux réserves minérales insolubles. Cette évolution peut être désignée par le terme de «mobilisation ». La mobilisation au niveau d'un horizon peut avoir deux origines différentes : elle peut, soit correspondre à un apport qui est dû à la décomposition des litières annuelles («cycle biologique » des éléments nutritifs), soit être due à une altération des réserves minérales de la roche mère. Pour reprendre les termes de Juste (1965) et Duchaufour (1968), nous parlerons de mobilisation biologique dans le premier cas, et de mobilisation chimique dans le second cas.

Pour les sols étudiés :

- dans le cas d'une mobilisation biologique, nous constatons généralement que l'apport annuel est relativement élevé par rapport à la quantité de l'élément considéré présente dans le sol. En outre, les horizons supérieurs humifères sont plus riches en cet élément que les horizons minéraux ;

- dans le cas d'une mobilisation chimique, l'apport annuel des litières en cet élément est négligeable par rapport à la quantité stockée dans le sol ; nous observons d'autre part une augmentation considérable de la teneur en cet élément vers les horizons minéraux profonds.

Cette notion de mobilisation de tel ou tel élément est particulièrement intéressante si nous prenons en considération les horizons $\mathbf{A}$ et $\mathbf{B}$ tels que nous les avons définis précédemment et que nous comparons leur teneur en cet élément, à l'apport de litière 
annuelle qui tombe sur le sol considéré à l'unité de surface. Pour prendre deux exemples types, celui du sol de La Costelle (MO 1), nous avons un rapport:

quantité de l'élément dans la litière annuelle

quantité de l'élément libre ou échangeable dans les horizons A et B

qui :

pour le potassium est de $1 / 40$

pour le calcium est de $1 / 10$

pour le magnésium est de 1/60

pour le phosphore $\left(\mathrm{P}_{2} \mathrm{O}_{5}\right)$ est de $1 / 130$

pour le fer est de $1 / 150000$

et pour l'aluminium de $1 / 70000$.

Alors qu'à la station de Bellefontaine (mc 1) (Forêt de Haye), ces rapports deviennent:

$\begin{array}{ll}\text { pour le potassium }: 1 / 25 \\ \text { pour le calcium }: 1 / 5000 \\ \text { pour le magnésium : } 1 / 200 \\ \text { pour le phosphore }: 1 / 25 \\ \text { pour le fer } & : 1 / 5000\end{array}$

Lorsque le rapport est élevé, rappelons (Duchaufour, 1968) que la mobilisation biologique tend à s'instaurer, le profil ayant tendance à s'enrichir aux dépens de la litière (cas du calcium dans les sols acides). Par contre, lorsque le rapport est faible, la mobilisation chimique l'emporte. C'est le cas du fer et de l'aluminium pour tous les sols étudiés, et aussi du calcium pour les sols calcaires (décarbonatation superficielle).

\section{2. - Etude comparative des caractéristiques du bilan}

\subsection{Variation $d u \mathrm{pH}$ des différents horizons $d u$ sol.}

Les $\mathrm{pH}$ des différents horizons de chacune de ces stations (à l'exception de celle de Fougères) ont été mesurés en septembre 1969, pendant une période de grande sécheresse. Les résultats sont intéressants, ils permettent de voir, comme le montrent les chiffres du tableau 2 et la figure 1 , que les $\mathrm{pH}$ des stations à mor et à moder croissent avec la profondeur (de 3,9 à 4,4 par exemple pour La Costelle MO 1), alors que ceux des stations à mull augmentent au contraire vers la surface (de 4,5 à 4,8 par exemple, pour la station des Hauts Bois mu 2) ; les $\mathrm{pH}$ des horizons $\mathrm{C}$ de ces différentes stations acides atteignent des valeurs assez voisines, comprises entre 4,1 et 4,5 .

2.2. Variation des teneurs en carbone, en azote et du rapport $C / N$ de ces différents horizons et de ces différentes stations.

Les teneurs en carbone et en azote des différents horizons sont variables d'une station à l'autre, mais il apparaît d'une façon nette, au vu de ces chiffres, que les valeurs correspondant aux teneurs en carbone et en azote des horizons A et B des sols à mor 


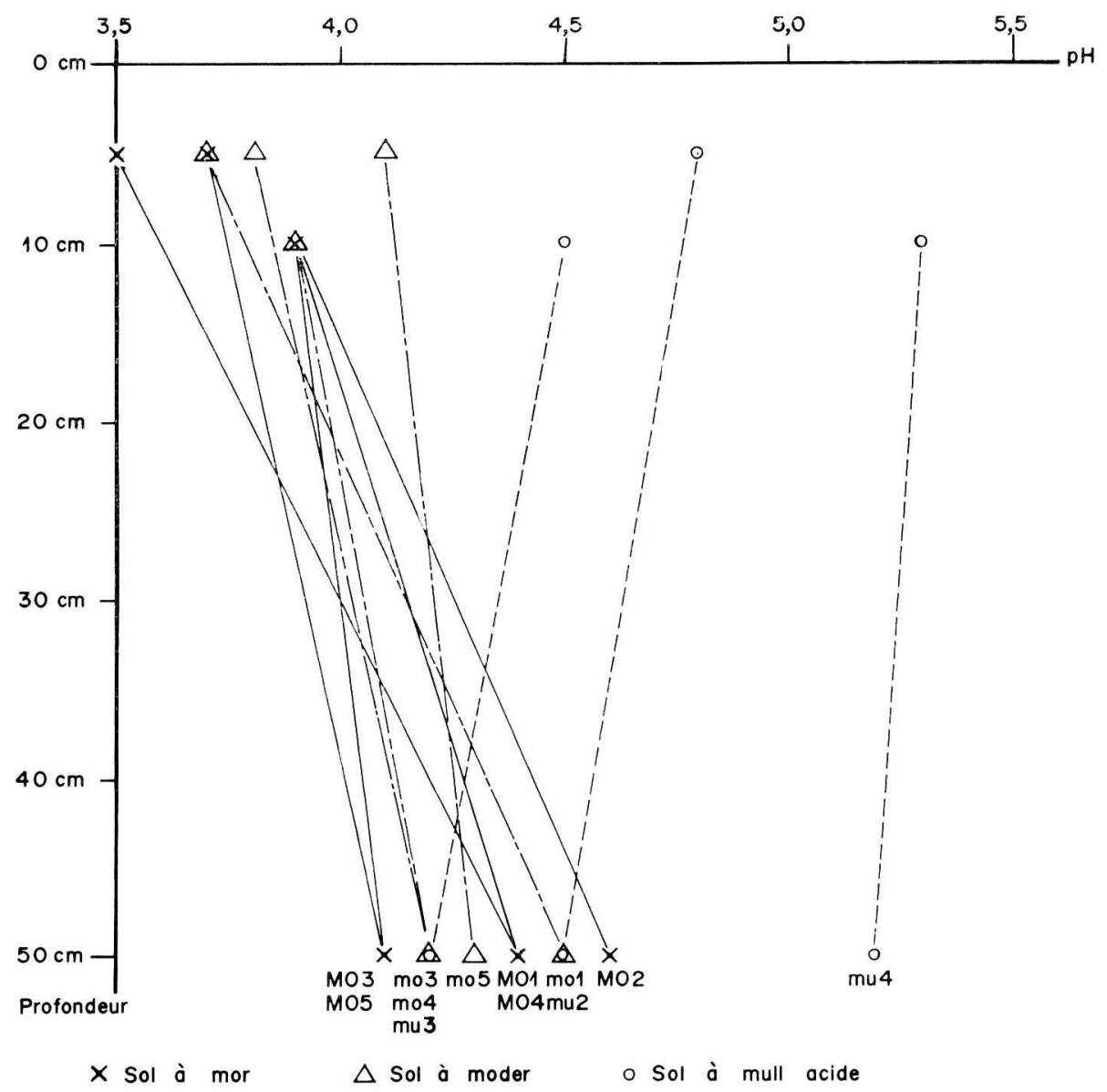

FIG. 1. - Variations du pH avec la profondeur pour les sols acides

et à moder, sont toujours beaucoup plus grandes que celles des sols à mull $(350 \mathrm{t}$ de $\mathrm{C} /$ ha en $\mathrm{A}$, soit $23 \mathrm{t} / \mathrm{cm}$ de sol à l'hectare et $350 \mathrm{t} /$ ha en $\mathrm{B}$, soit $8,7 \mathrm{t} / \mathrm{cm}$ de sol à l'hectare pour la station à mor de La Costelle MO 1, alors que pour le sol à mull des Hauts Bois mu 2, les valeurs correspondantes sont de $40 \mathrm{t} /$ ha soit $8 \mathrm{t} / \mathrm{cm}$ de sol en A, et $30 \mathrm{t} /$ ha, soit $0,75 \mathrm{t} / \mathrm{cm}$ de sol en $\mathrm{B}$ ).

Il en est de même de l'azote (11 t/ha soit $0,7 \mathrm{t} / \mathrm{cm}$ de sol en $\mathrm{A}, 12 \mathrm{t} / \mathrm{ha}$ soit $0,3 \mathrm{t} / \mathrm{cm}$ de sol en $\mathrm{B}$ à La Costelle MO 1 , et $2,5 \mathrm{t} /$ ha qui correspondent à $0,5 \mathrm{t} / \mathrm{cm}$ de sol en $\mathrm{A}$ et $3 \mathrm{t} /$ ha correspondant à $75 \mathrm{~kg} / \mathrm{cm}$ d'horizon $\mathrm{B}$ aux Hauts Bois mu 2).

Il y a donc un stockage de carbone et d'azote dans les horizons humifères des mor et des mull (en $A_{0}$ pour les mor, et en $A_{1}$ pour les mull), mais ce stockage est beaucoup plus important pour les mor que pour les mull; il est lié à la lenteur de la minéralisation et s'accompagne aussi d'une importante accumulation dans l'horizon B des sols à mor par migration des complexes organo-minéraux. 
Nous pouvons représenter, par un schéma très simple, les différents éléments du bilan. Si nous prenons le cas de l'azote (figure 2) dans quatre stations différentes : celle de La Costelle MO 1, celle de Bezange mo 2, celle du Climont mu 4 et celle de La Crédence mc 2, nous constatons d'abord, ce qui est évident puisque la roche mère ne contient pas d'azote, qu'il y a mobilisation biologique de l'azote (comme il y a une mobilisation biologique du carbone), mais aussi que les stockages de cet azote ont des importances très différentes. Il est très fort pour la station à mor, fort pour la station à moder, il reste encore élevé pour le mull calcique (surtout à cause de la présence des composés humiques qui sont stabilisés par le calcaire), alors qu'il est faible pour la station à mull acide où l'activité biologique est intense.

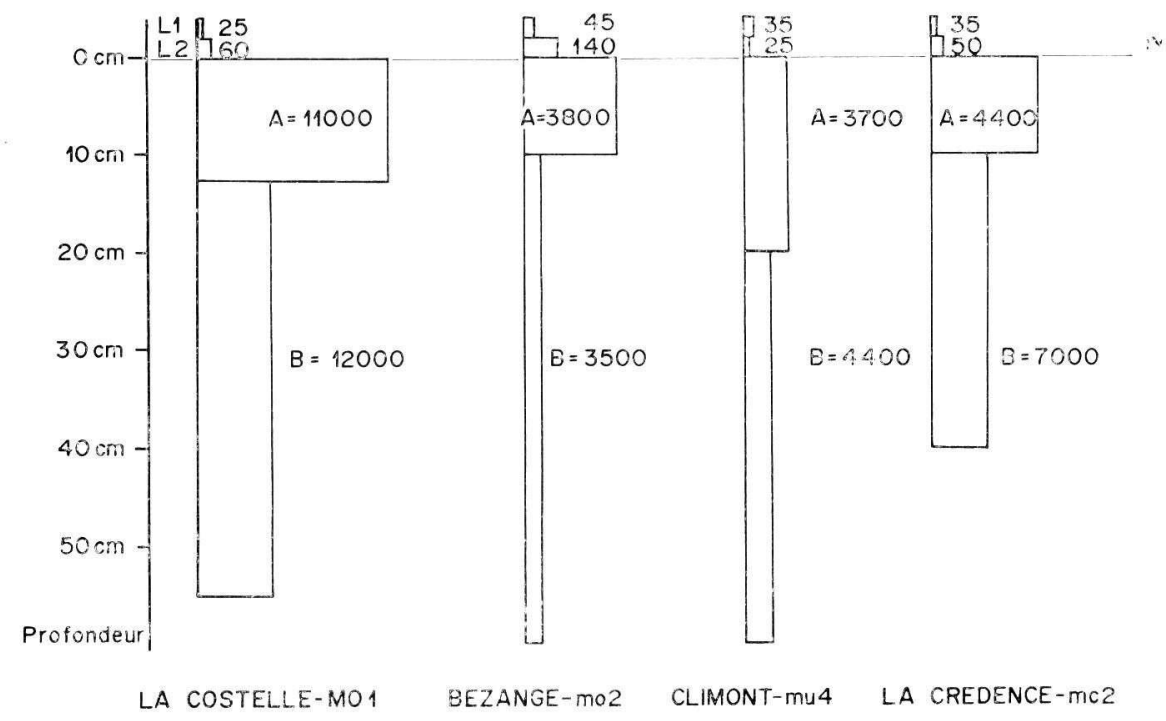

FIG. 2. - Bilan de l'azote dans différentes stations (en $\mathrm{kg} / \mathrm{ha}$ )

Le rapport $\mathrm{C} / \mathrm{N}$ diminue d'une façon régulière, dans toutes les stations, avec la profondeur où sa valeur reste d'ailleurs très variable (29 à La Costelle MO 1,11 à Basse-Landzoll MO 2 et 10 aux Hauts Bois mu 2), mais alors que dans les litières, les valeurs du rapport $\mathrm{C} / \mathrm{N}$ sont élevées et sans relation avec le type d'humus, à partir de l'horizon $\mathrm{A}_{1}$, les valeurs de $\mathrm{C} / \mathrm{N}$ caractérisent le type d'humus. La figure 3 montre comment décroît ce rapport, des litières frâ̂ches aux litières anciennes, puis au sol lui-même en $\mathbf{A}$ et en $\mathbf{B}$ pour les stations typiques. La courbe caractéristique des mor et des moder est une ligne brisée, alors que celle des mull correspond à une décroissance continue.

\subsection{Variation des teneurs en cations, de $S$, de $T$ et du rapport $S / T$.}

Rappelons tout d'abord que nous avons constaté dans la première partie de cette note que, pour ces sols acides, les apports annuels en divers éléments restent tous du même ordre de grandeur quelle que soit la station ; mais si nous comparons les quantités de cations présents à l'hectare dans chaque horizon au $\mathrm{cm}$ de sol, nous voyons 


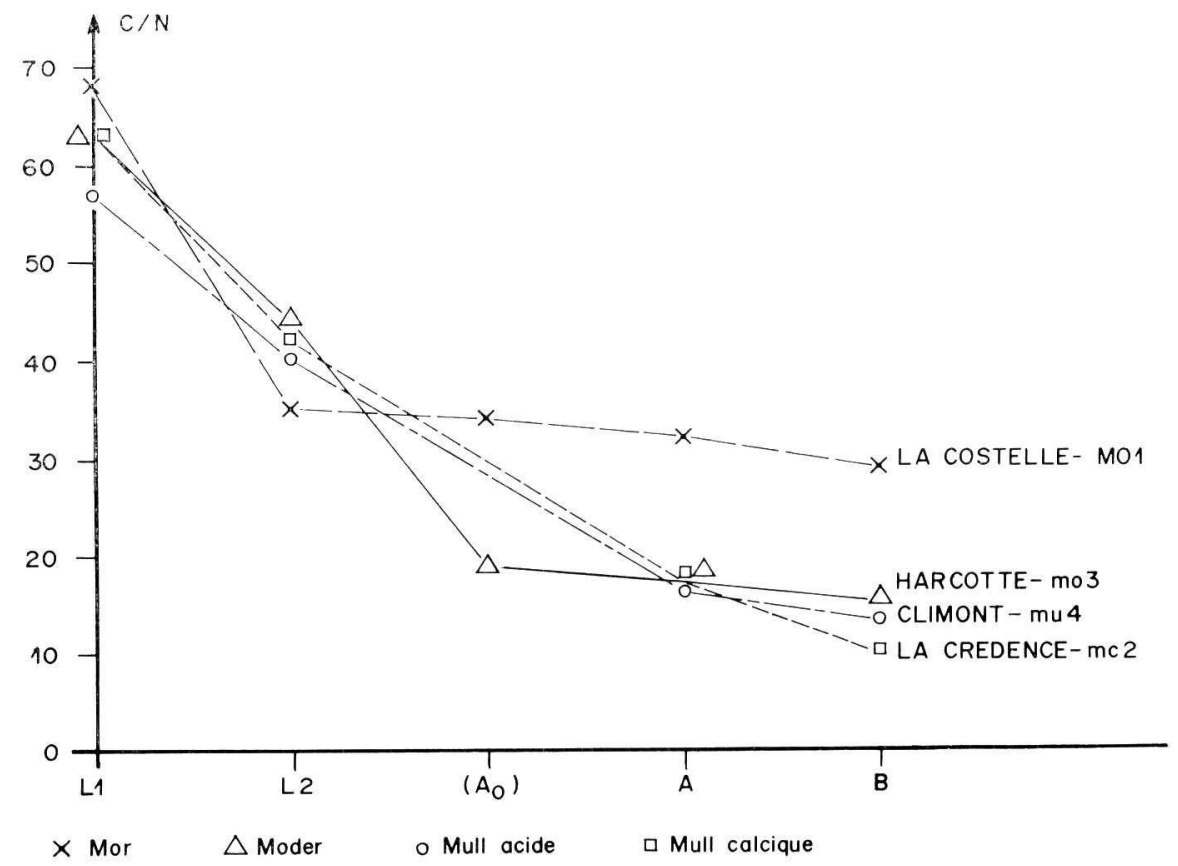

FIG. 3. - Variations du rapport $C / N$ dans différentes stations

que la somme des cations échangeables en A est toujours plus forte que celle présente en B. Il y a donc mobilisation biologique des cations échangeables alcalins et alcalinoterreux dans tous ces sols acides, quel que soit le type d'humus. Les variations d'un horizon à l'autre sont plus ou moins grandes et le stockage plus ou moins fort (le rapport $\frac{\mathrm{S} \text { de l'horizon } \mathrm{A}}{\mathrm{S} \text { de l'horizon } \mathrm{B}}$ est de 5 pour la station de Fougères MO 4, de 3,4 à La Costelle MO 1, de 2,9 aux Hauts Bois mu 2 et de 11 au Climont mu 4). Par contre, pour les sols calcaires, l'apport annuel dû à la litière est faible par rapport à l'énorme réserve préexistant dans le sol sous forme de carbonates, et il y a toujours mobilisation chimique. Pour illustrer ces considérations, nous représentons à la figure 4 les variations des teneurs en calcium échangeable pour une station à mor, une autre à moder, une à mull acide et une quatrième à mull calcique. Les apports annuels restant du même ordre de grandeur pour les trois premières stations, nous constatons qu'il y a un stockage considérable dans l'horizon A de La Costelle MO 1 (le rapport teneur en Ca échangeable par $\mathrm{cm}$ de $\mathrm{A} /$ teneur en $\mathrm{Ca}$ échangeable par $\mathrm{cm}$ de $\mathrm{B}$ est égal à 30), un stockage encore élevé dans l'horizon A du moder (le rapport précédent a une valeur de 5), alors qu'il est faible dans le mull acide (rapport $=1,7$ ) ; les valeurs absolues de la réserve en calcium échangeable dans tous les horizons A restent par ailleurs du même ordre de grandeur (de l'ordre de 200 à $300 \mathrm{~kg}$ de $\mathrm{Ca}$ échangeable/ha). Quant au mull calcique, bien que le sol reçoive chaque année sous forme de litière, de l'ordre de 2 fois plus de calcium que dans les autres stations, nous voyons qu'il y a une décarbonatation de l'horizon A par rapport à l'horizon $\mathrm{B}$ (le rapport précédemment défini devenant égal à 0,1 ). 


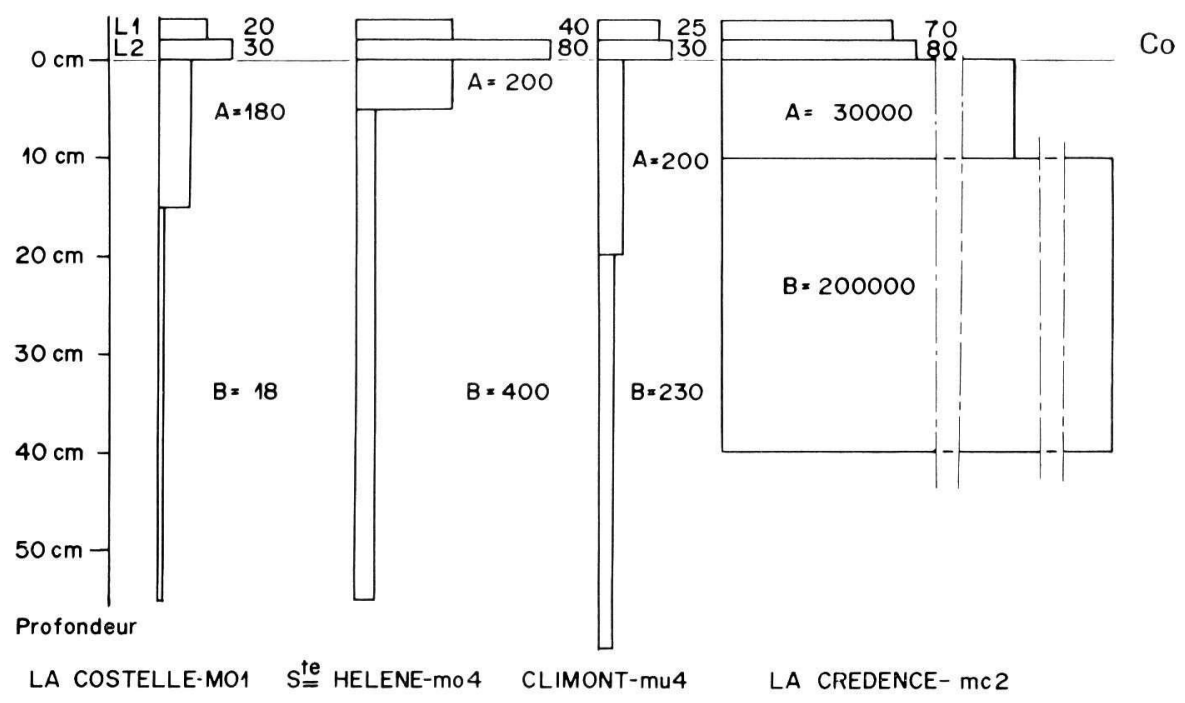

FIG. 4. - Bilan du calcium échangeable dans différentes stations (en $\mathrm{kg} / \mathrm{ha}$ )

Les valeurs de $\mathrm{T}$ sont assez variables d'un sol à l'autre ; elles sont plus fortes pour les sols à mor que pour les sols à mull (à cause de l'influence de la matière organique). Sur sols acides, ces valeurs de $\mathrm{T}$ sont toujours plus grandes pour l'horizon A (à cause de la capacité d'échange de la matière organique) que pour les horizons inférieurs. C'est ainsi qu'à La Costelle MO 1, dans l'horizon A, la valeur de T est de 32 m.eq./ $100 \mathrm{~g}$ de sol, en $\mathrm{B}$ elle est de $17 \mathrm{~m}$.eq./ $100 \mathrm{~g}$ de sol et qu'au Climont mu 4 , sur sol à mull, elle passe à $6 \mathrm{~m}$. eq. $/ 100 \mathrm{~g}$ en $\mathrm{A}$ et $4 \mathrm{~m}$.eq./100 $\mathrm{g}$ en $\mathrm{B}$.

Les taux de saturation $\mathbf{S} / \mathrm{T}$ de tous ces sols acides sont très faibles pour les sols à mor et à moder (inférieurs à $10 \%$ ) et apparemment un peu moins faibles pour les sols à mull. Cela va dans le sens des observations que nous venons de faire sur les valeurs de $\mathrm{T}$ (capacité d'échange de la matière organique). Dans les mor et les moder, le $\mathrm{S} / \mathrm{T}$ des horizons $\mathrm{A}$ est en général supérieur à celui des horizons $\mathrm{B}$ (influence de la matière organique), alors que dans les mull, il peut être plus faible, bien que ceci ne soit pas général (ce rapport $\frac{\mathrm{S} / \mathrm{T} \text { de } \mathrm{A}}{\mathrm{S} / \mathrm{T} \text { de } \mathrm{B}}$ est de 8 pour Fougères $\mathrm{MO} 4,3$ pour La Costelle MO 1, 1 pour Harcotte mo 3, 0,8 pour les Hauts Bois mu 2, mais de 9 pour SainteMarie mu 3). Les valeurs les plus faibles de ce rapport $S / T$ se rencontrent dans les sols à mull acide de montagne. Le lessivage climatique y est en effet fort important ; d'autre part, il ne faut pas oublier que l'horizon A de ces sols à mull, tel que nous l'avons défini, a été limité par l'allure de la courbe du carbone dans le profil ; il correspond donc à l'horizon $\mathrm{A}_{1}$ et à une partie plus ou moins importante de l'horizon $\mathrm{A}_{1} \mathrm{~B}$ de transition (les caractéristiques de l'horizon A sont donc des caractéristiques moyennes, et il est certain par exemple que le taux de saturation $\mathrm{S} / \mathrm{T}$ de l'horizon $\mathrm{A}$ est toujours inférieur au $\mathrm{S} / \mathrm{T}$ des premiers centimètres de l'horizon $\mathrm{A}_{1}$ ). 
Si nous comparons, pour une station donnée, les horizons organiques et les horizons minéraux, comme le $\mathrm{pH}$ des sols à mor et à moder augmente avec la profondeur alors qu'il diminue dans les sols à mull, nous voyons qu'il y a une corrélation négative entre le $\mathrm{pH}$ et le rapport $\mathrm{S} / \mathrm{T}$; dans les stations à mor et à moder, le rapport $\mathrm{S} / \mathrm{T}$ décroît de $\mathrm{A}$ vers $\mathrm{B}$, alors que le $\mathrm{pH}$ augmente, et dans les stations à mull, le rapport $\mathrm{S} / \mathrm{T}$ croît de $\mathrm{A}$ à $\mathrm{B}$, alors que le $\mathrm{pH}$ décroît.

Par contre, d'une station à l'autre, nous constatons qu'il n'y a aucune corrélation entre le rapport $\mathrm{S} / \mathrm{T}$ et le $\mathrm{pH}$.

Ceci prouve que pour ces sols très acides, le pH n'est pas lié aux teneurs en cations dits échangeables, tels que nous les dosons, mais dépend très probablement beaucoup plus de l'état de la matière organique (en particulier de la nature des acides organiques et de leur degré de dissociation) et aussi de la teneur en ions complexes hydroxy-alumineux dont le rôle, à ces $\mathrm{pH}$, est certainement très important.

\subsection{Variation des teneurs en sesquioxydes libres.}

Les apports annuels de fer et d'aluminium par les litières représentent une part infime de la masse totale du fer et de l'alumine libres des différents horizons inférieurs. Les rapports $\frac{\text { apport annuel }}{\text { réserve en } B}$ atteignent des valeurs comprises entre $1 / 60.000$ et $1 / 4.000$ pour l'aluminium, entre $1 / 120.000$ et $1 / 7.000$ pour le fer. La figure 5 montre comment se répartit l'alumine libre dans les horizons de différents types de sols. Nous voyons qu'il y a toujours mobilisation chimique, avec quelquefois une concentration très forte en $\mathrm{B}$ qui correspond au phénomène de podzolisation (comme à La Costelle MO 1).

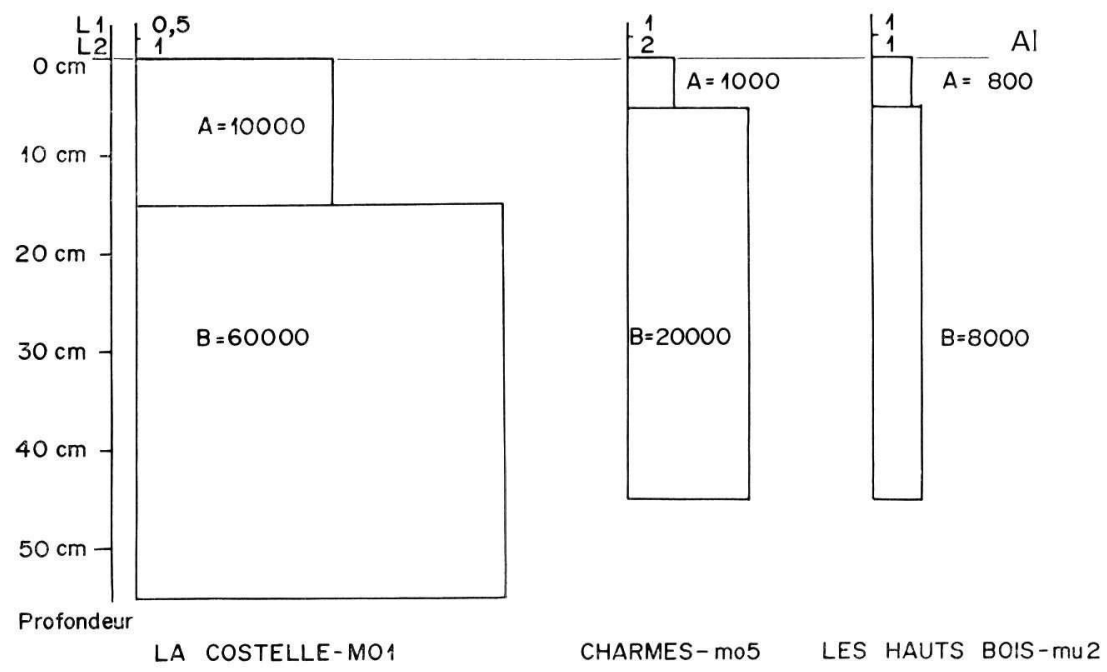

FIG. 5. - Bilan de l'alumine libre dans différentes stations (Al en $\mathrm{kg} / \mathrm{ha}$ ) 
Par contre, les apports annuels de silice par les litières, ne sont en général pas négligeables par rapport à la quantité totale de silice libre du sol. Les rapports sont de l'ordre de 1/10 et peuvent atteindre 1/4. La figure 6 montre qu'il y a tantôt mobilisation chimique, tantôt mobilisation biologique (tout spécialement dans les mull).

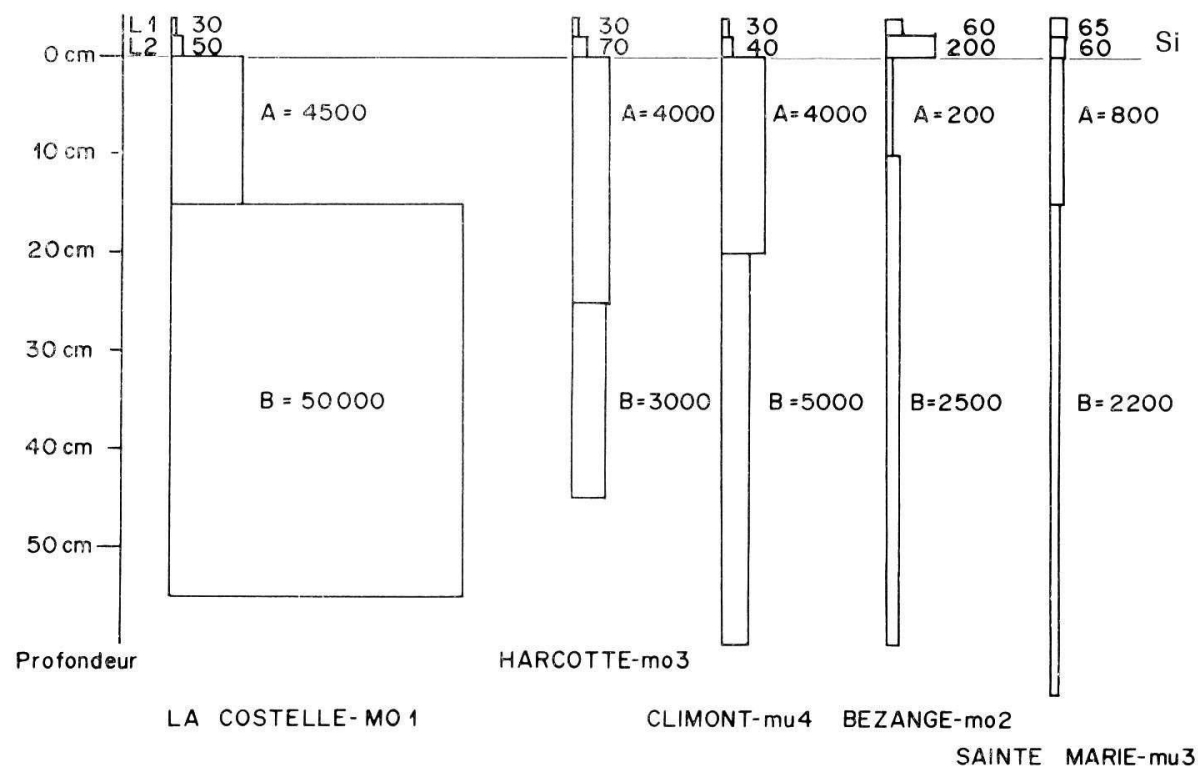

FIG. 6. - Bilan de la silice dans différentes stations (Si en $\mathrm{kg} / \mathrm{ha}$ )

\subsection{Variation des teneurs en $\mathrm{P}_{2} \mathrm{O}_{5}$ assimilable.}

Les apports annuels de phosphore total, eux aussi, ne sont pas négligeables, par rapport aux réserves des horizons sous-jacents. Ces apports peuvent représenter de $1 / 100$ à $1 / 10$ de la réserve en $\mathrm{P}_{2} \mathrm{O}_{5}$ assimilable des horizons $\mathrm{B}$. Les teneurs en $\mathrm{P}_{2} \mathrm{O}_{5}$ varient d'une station à l'autre de 1 à 10 ; mais les variations à l'intérieur d'une même station sont beaucoup plus intéressantes, comme le montre la figure 7 où il apparaît qu'il y a, comme dans le cas de la silice libre, mobilisation chimique pour les sols à mor et à moder, et mobilisation biologique pour les sols à mull acide et à mull calcique.

\section{CONCLUSION}

L'intérêt des chiffres présentés dans cette note, tient essentiellement au fait que les stations étudiées sont toutes des Hêtraies dépourvues de sous-bois et que les roches mères (à l'exception des deux stations à mull calcique) sont toutes très acides. Dans de telles conditions, nous constatons :

$1^{\circ}$ que les litières amènent chaque année à peu près la même quantité d'éléments dans toutes les stations; 


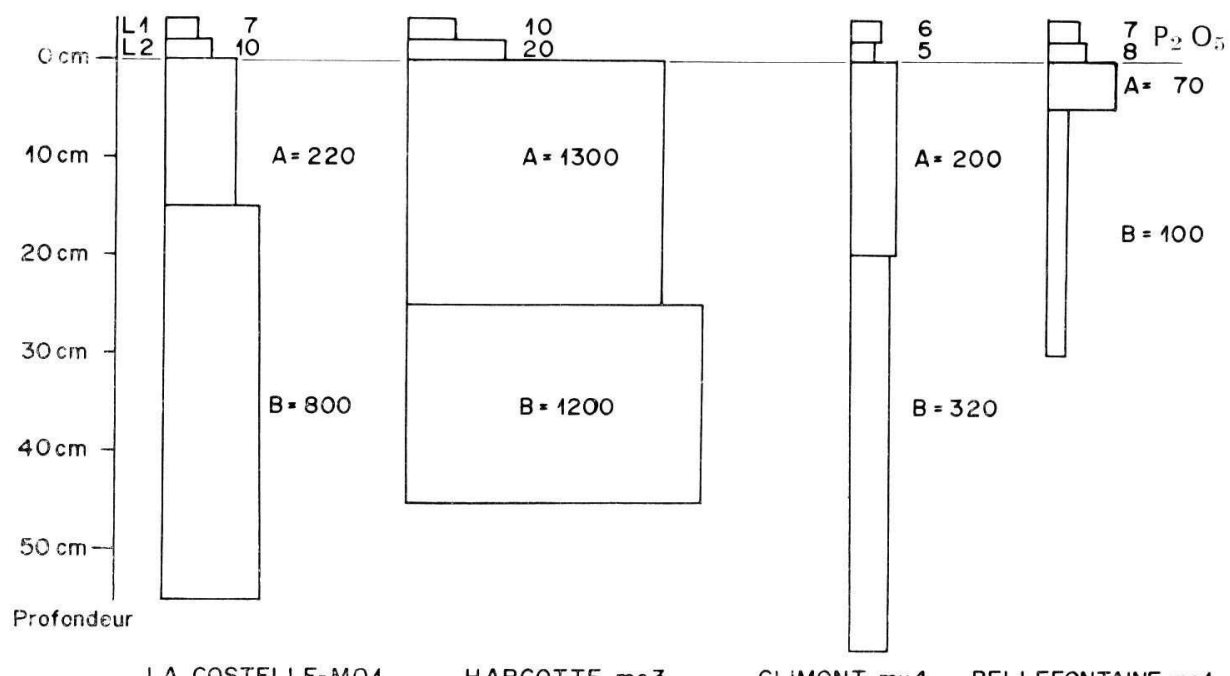

FIG. 7. - Bilan du phosphore dit assimilable dans différentes stations $\left(P_{2} O_{5}\right.$ en $\left.\mathrm{kg} / \mathrm{ha}\right)$

$2^{\circ}$ que les $\mathrm{pH}$ de ces sols varient en profondeur d'une façon différente sous les mull et sous les mor ;

$3^{\circ}$ que les $\mathrm{C} / \mathrm{N}$ décroissent régulièrement des litières vers les horizons de profondeur, et qu'il y a un stockage de l'azote et du carbone en $\mathrm{A}$ et en B plus grand dans les mor que dans les mull;

$4^{\circ}$ que le stockage des bases est sensiblement le même dans les mull et dans les mor, et qu'il est toujours plus fort dans les horizons A que dans les horizons B ;

$5^{\circ}$ que la somme des bases échangeables (telle que nous la mesurons actuellement) ne présente pas de corrélation avec le $\mathrm{pH}$, pas plus d'ailleurs que le taux de saturation n'en présente avec le $\mathrm{pH}$;

$6^{\circ}$ qu'il y a, pour toutes les stations, une mobilisation biologique du carbone et de l'azote, pour les stations acides seulement, une mobilisation biologique des cations échangeables, une mobilisation chimique pour le fer et l'aluminium, mais qu'il y a tantôt mobilisation biologique (apparemment dans le cas des mull), tantôt une mobilisation chimique (apparemment dans le cas des mor et des moder) pour la silice et le phosphore dit assimilable.

Toutes ces constatations ont leur importance mais, si elles ne sont pas généralisables à d'autres zones de $\mathrm{pH}$, elles permettent au moins de ramener à leur juste importance certains facteurs qui ont été quelquefois décrits, comme essentiels, dans le déterminisme des types d'humus: on peut affirmer en particulier que, sur roche mère acide et pauvre en bases (comme les granites ou les grès), ce n'est pas l'apport annuel de cations et d'azote qui est l'agent déterminant du type d'humus (mull, moder ou mor). Il semble que d'autres facteurs interviennent, en particulier, le «pédoclimat» et ses variations saisonnières. De nouvelles études, actuellement en cours, abordent cet important problème. 


\title{
SUMMARY \\ A COMPARATIVE STUDY OF THE BALANCES OF PLANT NUTRIENTS IN SOME BEECH FORESTS SOILS
}

The present paper gives an account of the balance of plant nutrients in some acidiphile Beech forests in Eastern France (in order to make a comparison, two Beech forests on rendzina have been also studied). The amounts of plant nutrients returning each year to the forest soil at the leaf fall are compared with the quantity of elements preexisting in the soil as an exchangeable form; so that it is possible to distinguish two different types of elements'mobilization.

1) A biological mobilization (for carbon, nitrogen, calcium) giving an accumulation of the nutritive elements in the upper horizons ( $A_{1}$ for a mull, $A_{0}$ for a mor).

2) A chemical mobilization (for aluminium and iron) giving an accumulation of the nutritive elements in the underlying mineral soils by an alteration's process. Both silica and phosphorus may undergo either one or the other type of mobilization.

In conclusion, the quantity of nutritive element annually turning back to the soil by the leaf fall presents only small variations comparatively to the variations of the parent rock, and has a minor part in the determinism of the humus type.

\section{ZUSAMMENFASSUNG}

\section{VERGLEICHENDE UNTERSUCHUNGEN DER NAHRSTOFFBILANZEN VON GEWISSENEN}

\author{
BUCHENWALDERBODEN
}

Der Ziel dieses Arbeit ist die Bestimmung des Nährstoffbilanzes unter gewissene Büchenwälder Ost-Frankreichs (zwei Buchenwälder auf Rendzina wurden auch untersucht zum Vergleich). Der jährliche Zufuhr der verschiedenen Elemente durch das Streu wird mit der Menge dieser im Boden unter mobiliserbarer Form vorhandenen Elemente vergliechen. So ist es möglich zwei Freisetzungsprozessen dieser Elemente festzustellén :

1) eine biologische Freisetzung (Kohlenstoff, Stickstoff und Calcium betreffend) die eine Anreicherung der Elemente in den humosen Horizonten auswirkt (im $\mathrm{A}_{1}$ Horizont wenn es sich um einen Mull handelt, und im $\mathbf{A}_{0}$. Horizont im Fall eines Rohhumus).

2) eine chemische Freisetzung (für Aluminium und Eisen) die eine Verlagerung der Elemente in den Mineralhorizonten durch Verwitterungsprozess verursacht. Silicium und Phosphor können an beiden Freisetzungen teilnehmen.

Schliesslig, unterliegt der Anteil der Nährstoffe, die jährlich zum Boden mit dem Streu zurückkehren, nur schwache Schwankungen, im Vergleich zu der Zusammensetzung der Ausganggesteine und spielt nur eine geringe Rolle in der Entstehung der Humusform. 


\section{REFERENCES BIBLIOGRAPHIQUES}

Coulson C. B., Davies R. J., Lewis D. A., 1960. J. soil sci., 11 (1), 20 (1), 30 (2).

Duchaufour Ph., 1965. Précis de Pédologie. Masson et $\mathrm{C}^{\mathrm{ie}}$, éd., Paris.

Duchaufour Ph., 1968. L'évolution des sols. Essai sur la dynamique des profils. Masson \& $\mathrm{C}^{\mathrm{ie}}$, éd. Paris.

Duchaufour Ph., Bonneau M., 1959. Une méthode nouvelle de dosage du phosphore assimilable dans les sols forestiers. Bull. A.F.E.S., 4, 1959, 193-198.

Duchaufour Ph., Souchier B., 1966. Note sur une méthode d'extraction combinée de l'aluminium et du fer libres dans les sols Sci. Sol, (1), $1^{\text {er }}$ sem. 1966.

JACQUiN F., 1963. Thèse Fac. Sci., Nancy, 156 p.

Juste C., 1965. Thèse Fac. Sci. Nancy, 143 p.

RuHL A., 1957. Eine Auswertung von Streudnalysen aus nordeutschen Mittelgebirgen. Forstm. Centralblatt, 11-12, 364-376.

Toutain F., 1966. Etude du sol et des eaux de la forêt de Fougères. Thèse de doctorat de $3^{\text {e }}$ cycle. Faculté des sciences de Paris. 